\title{
IR hot carrier based photodetection in titanium nitride oxide thin film- Si junctions
}

\author{
Nicholas A. Güsken ${ }^{1, *}$, Alberto Lauri ${ }^{1}$, Yi Li ${ }^{1,3}$, Andrea Jacassi, ${ }^{1}$ Takayuki Matsui ${ }^{1}$, Brock Doiron ${ }^{1}$, Ryan Bower ${ }^{2}$, Anna \\ Regoutz $^{2}$, Andrei Mihai ${ }^{2}$, Peter K. Petrov ${ }^{2}$, Rupert F. Oulton ${ }^{1}$, Lesley F. Cohen ${ }^{1}$, Stefan A. Maier ${ }^{1,3}$ \\ ${ }^{1}$ The Blackett Laboratory, Department of Physics, Imperial College London, London SW7 2AZ, United Kingdom. \\ ${ }^{2}$ Department of Materials, Imperial College London, London SW7 2AZ, United Kingdom. \\ ${ }^{3}$ Chair in Hybrid Nanosystems, Nanoinstitute Munich, Fakultät für Physik, Ludwig-Maximilians Universität München, 80539 München, \\ Germany.
}

*Correspondence: n.gusken16@imperial.ac.uk

\begin{abstract}
:
Hot carrier based methods constitute a valuable approach for efficient and silicon compatible sub-bandgap photodetection. Although, hot electron excitation and transfer have been studied extensively on traditional materials such as Au and Ti, reports on alternative materials such as titanium nitride (TiN) are rare. Here, we perform hot hole photodetection measurements on a p-Si/metal thin film junction using Ti, Au and TiN. This material is of interest as it constitutes a refractory alternative to Au which is an important property for plasmonic applications where high field intensities can occur. In contrast to Au, a TiN/Si junction does not suffer from metal diffusion into the Si, which eases the integration with current Si-fabrication techniques. This work shows that a backside illuminated p-Si/TiN system can be used for efficient hot hole extraction in the IR, allowing for a responsivity of $1 \mathrm{~mA} / \mathrm{W}$ at an excitation wavelength of $1250 \mathrm{~nm}$ and at zero bias. Via a comparison between TiN and other commonly used materials such as Au, the origin of this comparably high photoresponse can be traced back to be directly linked to a thin $\mathrm{TiO}_{2-x}$ interfacial layer allowing for a distinct hot-hole transfer mechanism. Moreover, the fabrication of TiN nanodisk arrays is demonstrated which bears great promise to further boost the device efficiency.
\end{abstract}

\section{INTRODUCTION:}

Novel approaches enabling efficient ways to create photo-voltages, range from 2D material plasmonic hybrid structures [1-3] over metal-insulator-metal waveguides [4,5] to semiconductor/metal nanowire junctions [6]. One of the most widely studied systems for hot-carrier based photodetection however, are common semiconductor(SC)/metal junctions. Indeed, this type of hot carrier photo-detection platform has attracted enormous attention mainly due to its capability of creating a photovoltage at a semiconductor/metal junction without being limited by the semiconductor bandgap energy. Additionally, the ability to pattern junctions allowing for plasmonic resonances, enabled a strong efficiency boost for this type of device. In fact, collective charge oscillations in form of plasmonic resonances give rise to highly energetic hot-carrier distributions at the semiconductor interface and allow to significantly increase carrier collection efficiencies. In spite of studying the well discussed impact of plasmonic resonant structures on hot-carrier excitation, the work presented here [7] explores the use of TiN thin films. This material is of particular interest as it constitutes a refractory and non-diffusive alternative to other materials more commonly used in plasmonic applications. Indeed, resonantly excited plasmonic structures lead to strong fields in the vicinity to the metal surface which gives rise to energetic and hot carrier distributions inside the metal. The excited carriers can then be easily extracted in order to generate a strong photocurrent. Due to TiN's valuable properties including bio-compatibility, mechanically robustness as well as tuneable oxygen content, it represents a promising candidate for Si-compatible hot-carrier photo-detection devices.

In addition, many studies in the community explored the excitation, transport and collection mechanisms of hot electrons across Schottky junctions. However, the role and use of hot holes has not yet been studied extensively. While some recent studies predict an increase in extraction efficiency compared to their hot electron counterparts [1], only a few experimental studies [8] exploring the underlying transport properties exist. 


\section{EXPERIMENT, RESULTS \& DISCUSSION:}

Here [2], we perform hot hole photodetection measurements on a p-Si/metal thin film junction comparing various metals such as Ti, Au and TiN. A backside illuminated p-Si/TiN Schottky junction gives rise to strong thin film absorptions, constituting an ideal testbed for this type of measurements. Such a simple back-side illumination configuration of a thin film Schottky device has been demonstrated to be an efficient solution for hot carrier excitation [9]. The measurement configuration is illustrated in Figure 1a) illustrating the electrically contacted junction. Figure 1b) shows the temporal photoresponse of $\mathrm{p}$-Si/Au compared to a $\mathrm{p}$-Si/TiN sample, while the laser is turned on and off consecutively. A strong responsivity of $1 \mathrm{~mA} / \mathrm{W}$ at an excitation wavelength of $1250 \mathrm{~nm}$ and at zero bias is achieved for the TiN sample, which is in the competitive range with comparable zero-bias photodetection studies at this wavelength [9]. Moreover, this response is above one order of magnitude stronger than in the $\mathrm{p}$-Si/Au case while the absorption only differs by a factor of 2 as shown below. Note that TiN exhibits a few nanometres thin $\mathrm{TiO}_{2-x}$ layer at its surface. $\mathrm{TiO}_{2-x}$ exhibits distinct inter-bandgap trap states and can alter the hot-electron injection behaviour in $\mathrm{Au}-\mathrm{TiO}_{2}$ systems [10]. In order to get to the bottom of the different efficiencies, a range of comparable samples was investigated, for which an additional $5 \mathrm{~nm}$ thin $\mathrm{TiO}_{2-\mathrm{x}}$ has been introduced via sputtering.

Figure 1c) shows the photoresponsivity dispersion of a range of samples in the near-IR. It becomes obvious that the $\mathrm{TiO}_{2-\mathrm{x}}$ containing samples show a very distinct response and an enhanced photoresponsivity between $1200 \mathrm{~nm}$ and 1400 $\mathrm{nm}$ compared to the non-oxide containing system.
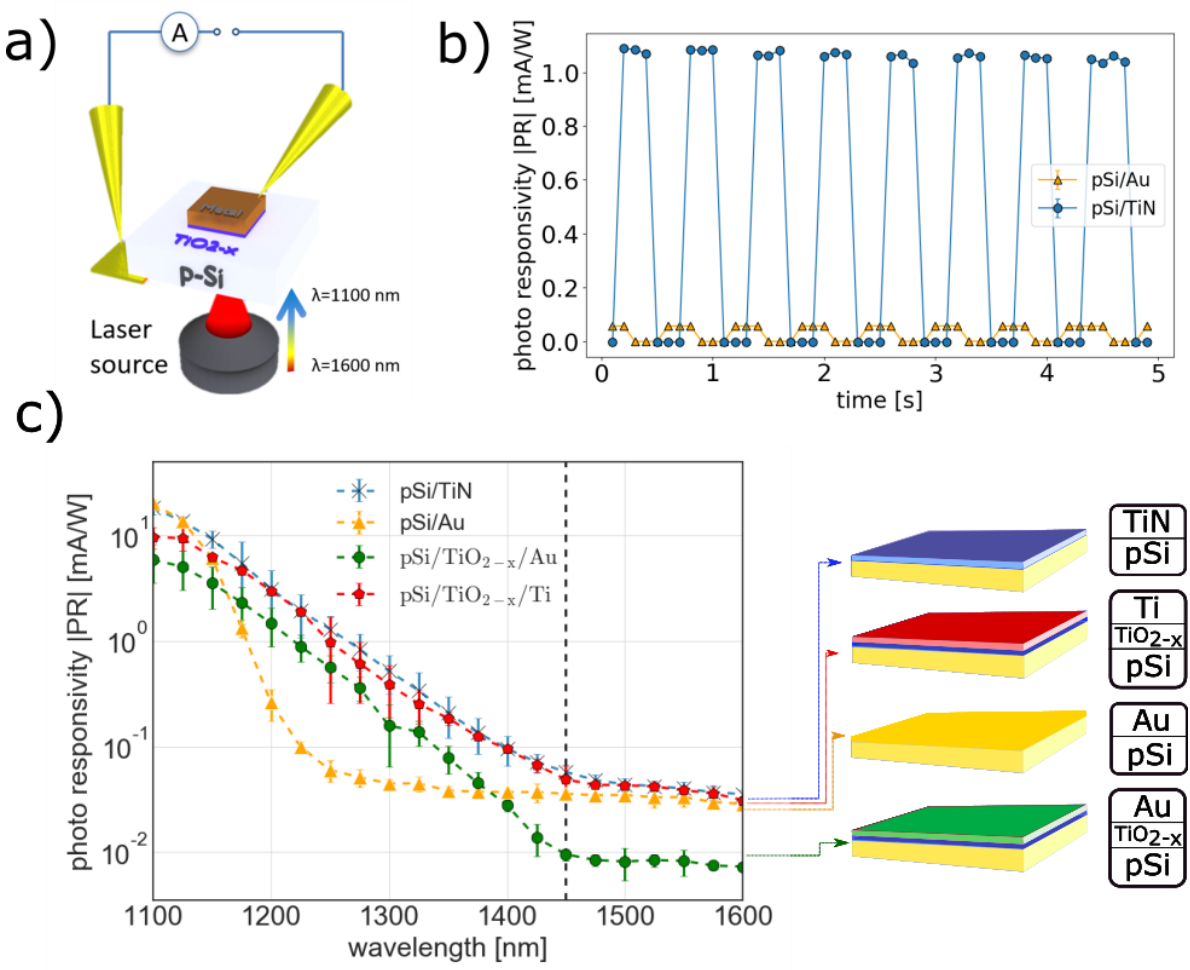

Figure 1: a) Schematic of the measurement configuration showing a wavelength tuneable laser source and a backside illuminated, electrical contacted $\mathrm{p}$-Si/absorber junction. b) temporal photoresponse of a p-Si/TiN junction compared to a $\mathrm{p}-\mathrm{Si} / \mathrm{Au}$ junction without applied bias. c) Photoresponsivity dispersion according to various junctions shown schematically on the right-hand side.

The deviation is not due to any resonant absorption features as shown by the flat response of absorption measurements conducted on the same samples.[7] Taking the absorption of the individual systems into account. The internal quantum efficiency (IQE) can be determined as shown in Figure 2. Here, the similarity of the signal amongst the TiO2-x containing samples exhibiting a distinct response compared to the $\mathrm{p}$-Si/Au system can be confirmed. Interestingly, the IQE seems almost independent of the metal absorber used which hints towards the fact that not the metal/TiO2-x interface is the determining factor of the differing response but rather the $\mathrm{p}-\mathrm{Si} / \mathrm{TiO} 2-\mathrm{x}$ interface which is the same across all oxide containing junctions. Moreover, a distinct cut-off at a wavelength of $1450 \mathrm{~nm}$ can be 
observed for all oxide containing samples, at which the photoresponse strongly changes its dispersion. This behaviour implies that the excited carriers encounter a very similar energy barrier independently of the metal-oxide combination used, which has been

Turning the attention to the interface composition, a few nanometer thick amorphous $\mathrm{TiO}_{2-x}$ layer forms during the growth process. Despite the fact that, based on a bandgap energy of about $3.2 \mathrm{eV}, \mathrm{TiO}_{2-\mathrm{x}}$ is expected to act as a hole blocking layer, amorphous $\mathrm{TiO}_{2-x}$ allows for hole carrier transfer via trap states [11], which consist of $\mathrm{Ti}^{3+}$ states and oxygen vacancies. Thus, we propose a trap state mediated carrier transfer mechanism which is supported by our results obtained via optical and electrical transport measurements. A simplified band model diagram outlining a possible transfer of hot holes from the metal via the trap states into the p-Si is shown in Figure 3a). The built-in potential $\left(\mathrm{V}_{\mathrm{bi}}\right)$ was estimated based on the dark IV measurements as explained above.

Based on the results presented we come to the conclusion that a few nanometres thin $\mathrm{TiO}_{2-\mathrm{x}}$ interlayer facilitates the hot hot-hole transfer and injection from the metal absorber into the p-type semiconductor collector via trap states. This

a)

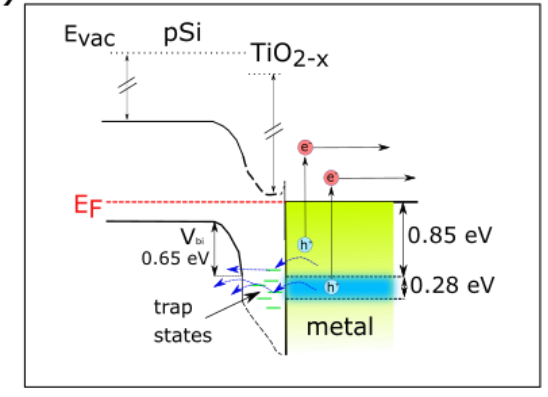

b)

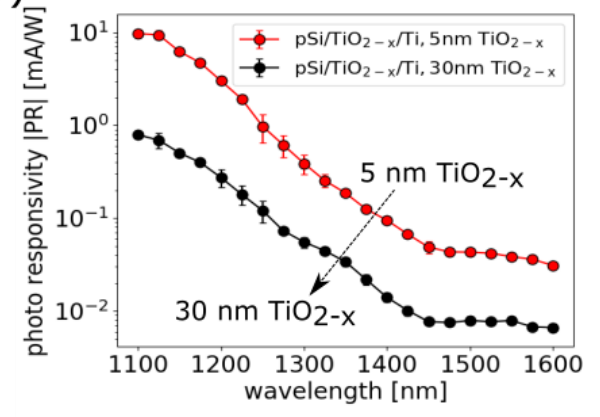

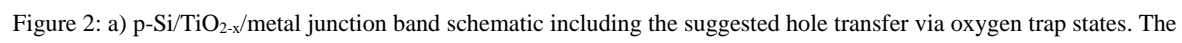
blue area in the metal highlights the strongly photoresponsive regime. b) $\mathrm{PR}$ dispersion of $\mathrm{p}-\mathrm{Si} / \mathrm{TiO}_{2-\mathrm{x}} / \mathrm{Ti}$ for a $5 \mathrm{~nm}$ and $30 \mathrm{~nm} \mathrm{TiO}_{2-\mathrm{x}}$ interlayer, respectively.

claim is additionally supported by XPS measurements showing trap states signatures [7]. Moreover, we have tested different thicknesses of the oxide interlayer as it can be expected that the transport is heavily dependent on the depletion region width at the boundary to the $\mathrm{TiO}_{2-\mathrm{x}}$ which is about $9 \mathrm{~nm}$ wide, as well as recombination taking place within the usually n-type oxide. Figure $3 \mathrm{~b}$ ) demonstrates that the photoresponsivity decreases by over an order of magnitude when enlarging the $\mathrm{TiO}_{2-x}$ interlayer from $5 \mathrm{~nm}$ to $30 \mathrm{~nm}$, which is in line with this picture. Moreover, it supports that the signal observed is not resulting from a simple trap state photo-excitation process within the oxide layer.

Finally, the attention is turned to the fabrication of TiN nanoparticles and antennas. Plasmonic nanostructures enable to exploit plasmonic resonances which allow to enhance the photoresponse even further, when used as the metal side of
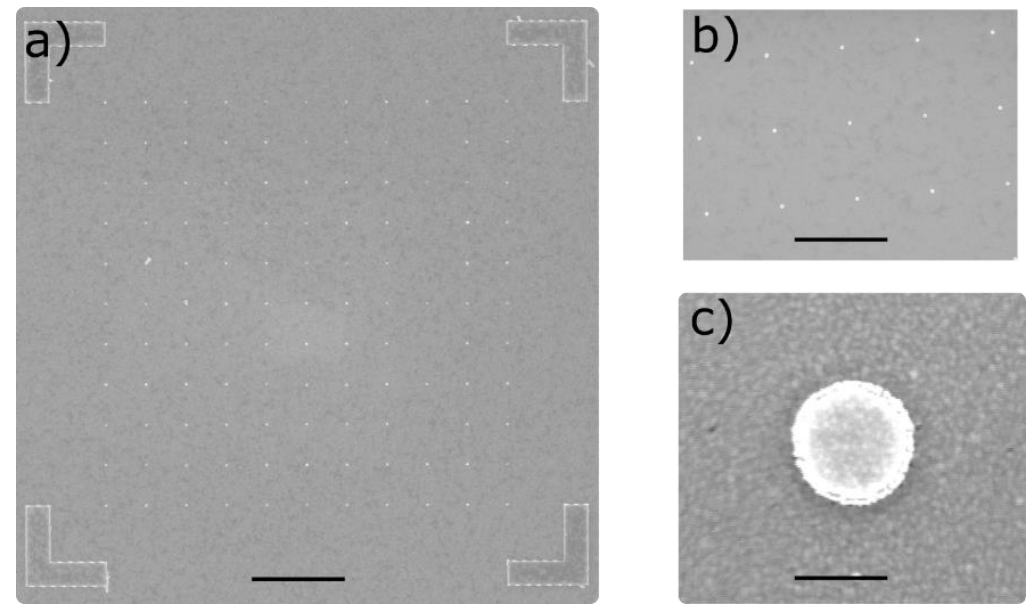

Figure 3: Structured TiN spheres. a) Overview of a TiN array, scale bar $10 \mu \mathrm{m}, \mathrm{b}$ ) close view, scale bar $4 \mu \mathrm{m}$ and c) close up showing a single TiN sphere, scale bar $150 \mathrm{~nm}$. The film thickness is $40 \mathrm{~nm}$. 
the Schottky junction. This is due to a large hot carrier density of states resulting from the strong sub-wavelength light confinement in close vicinity to the surface of the resonant structures. The patterning of TiN is in particular challenging due to its chemical characteristics which make this material a robust and non-refractive alternative to Au and Ag.

We demonstrate the fabrication of TiN disk arrays as shown in Figure 4a)-c) based on a 7 step process. First, TiN is deposited via Plasma Enhanced Chemical Vapor Deposition (PECVD), the sample is then spin coated with an electron sensitive resist, e.g. PMMA. The disk array design is written into the polymer with Electron Beam Lithography (EBL) followed by a development step in IPA. A $30 \mathrm{~nm}$ Cr layer is evaporated onto the patterned and developed resist, acting as hard mask for the dry etching. The residual PMMA is removed with a lift off in acetone and the redundant TiN is removed via Reactive Ion Etching (RIE); the Cr mask protects the TiN from being removed in the patterned area. As a last step, wet etching with $\mathrm{Cr}$ etchant is performed to remove the $\mathrm{Cr}$ mask from the sample, resulting in the TiN disk array shown in Figure 4a)-c). This approach allows to access a minimum feature size of $60 \mathrm{~nm}$ which accords to resonances at far shorter wavelength then the IR regime.
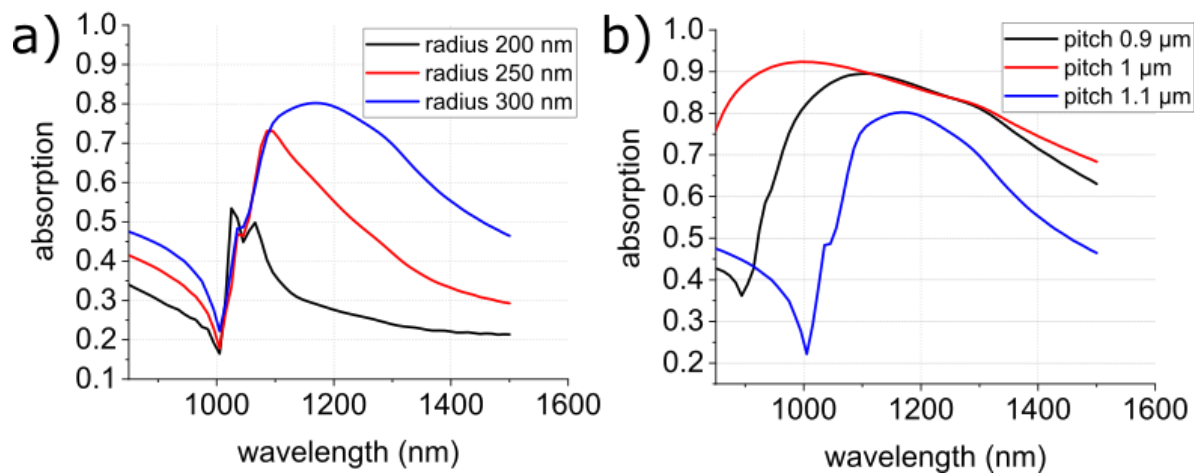

Figure 5: FDTD simulations of TiN disk arrays for a) different radii of the disks at a constant pitch of $1 \mu \mathrm{m}$ and b) for different pitch values at a constant disk radius of $300 \mathrm{~nm}$. A TiN film thickness of $40 \mathrm{~nm}$ was used in the simulation.

Hence, the disk radius and the pitch of the disk array can be chosen such that plasmonic resonances occur in the IR region of the spectrum. Different array pitches and radii of the TiN particles were simulated in order to optimize the absorption between $1.1 \mu \mathrm{m}$ and $1.5 \mu \mathrm{m}$, shown in Figure 5a)-b). The results show that TiN disks of $300 \mathrm{~nm}$ radius and an array pitch of $1 \mu \mathrm{m}$ are the most suitable one for the use in the IR, exhibiting up to $90 \%$ absorption.

\section{CONCLUSION:}

This work shows that TiN thin films on p-Si can be used for comparably efficient self-powered and Si-compatible photocurrent generation in the Si sub-bandgap infrared region. Measurements on a $50 \mathrm{~nm}$ TiN film on p-Si yield a photoresponsivity which is about one order of magnitude higher compared to $\mathrm{p}-\mathrm{Si} / \mathrm{Au}$, resulting in a peak value of 1 $\mathrm{mA} / \mathrm{W}$ at $1250 \mathrm{~nm}$. Our results unambiguously demonstrate that a few nanometres thin $\mathrm{TiO}_{2-\mathrm{x}}$ interfacial layer forming during TiN growth at the Si/TiN interface allows for an increase in photoresponsivity of about one order of magnitude, compared to a clean $\mathrm{p}-\mathrm{Si} /$ metal interface. Moreover, in conjunction with $\mathrm{p}-\mathrm{Si}$, such thin layer of interfacial $\mathrm{TiO}_{2-\mathrm{x}}$, can be used as a platform for photocurrent enhancement in combination with a range of different metal films. The direct comparison between $\mathrm{p}-\mathrm{Si} / \mathrm{Au}$ and $\mathrm{p}-\mathrm{Si} / \mathrm{TiO}_{2-\mathrm{x}} / \mathrm{Au}$ photocurrent responses at zero bias, further underpins this observation, outlining that the photoresponse dispersion relation of a $\mathrm{TiO}_{2-\mathrm{x}}$ thin film containing system clearly deviates from the response of a $\mathrm{p}-\mathrm{Si} / \mathrm{metal}$ system. Indeed, we find that the introduction of an oxide interlayer allows for conversion of the photo dispersion from a Fowler-like behaviour, observed in an oxide-free system, into a p-Si/TiN-like response, observed in the oxide-containing system. Moreover, the patterning of TiN plasmonic resonant structures has been demonstrated which allows to boost the efficiency of the presented junction even further. 


\section{REFERENCES:}

[1] V. Shautsova, T. Sidiropoulos, X. Xiao, N.A. Güsken, N.C.G. Black, A.M. Gilbertson, V. Giannini, S.A. Maier, L.F. Cohen, and R.F. Oulton, Nat. Commun. 9, 5190 (2018).

[2] V. Shautsova, N.A. Güsken, T. Sidiropoulos, X. Xiao, N.C.G. Black, A.M. Gilbertson, V. Giannini, S.A. Maier, L.F. Cohen, and R.F. Oulton, in Conf. Lasers Electro-Optics (OSA, Washington, D.C., 2019), p. FTh3C.5.

[3] F.H. Koppens, T. Mueller, P. Avouris, a C. Ferrari, M.S. Vitiello, and M. Polini, Nat Nanotechnol 9, 780 (2014).

[4]Y. Salamin, P. Ma, B. Baeuerle, A. Emboras, Y. Fedoryshyn, W. Heni, B. Cheng, A. Josten, and J. Leuthold, ACS Photonics 5, 3291 (2018).

[5] N.A. Güsken, M.P. Nielsen, N.B. Nguyen, S.A. Maier, and R.F. Oulton, Opt. Express 26, 30634 (2018).

[6] M. Sistani, M.G. Bartmann, N.A. Güsken, R.F. Oulton, H. Keshmiri, M.S. Seifner, S. Barth, N. Fukata, M.A. Luong, M.I. den Hertog, and A. Lugstein, Appl. Phys. Lett. 115, 161107 (2019).

[7] N.A. Güsken, A. Lauri, Y. Li, T. Matsui, B. Doiron, R. Bower, A. Regoutz, A. Mihai, P.K. Petrov, R.F. Oulton, L.F. Cohen, and S.A. Maier, ACS Photonics acsphotonics.8b01639 (2019).

[8] M. Tanzid, A. Ahmadivand, R. Zhang, B. Cerjan, A. Sobhani, S. Yazdi, P. Nordlander, and N.J. Halas, ACS Photonics 5, 3472 (2018)

[9] L. Krayer, E.M. Tennyson, M.S. Leite, and J.N. Munday, ACS Photonics acsphotonics.7b01021 (2017).

[10] E. Blandre, D. Jalas, A.Y. Petrov, and M. Eich, ACS Photonics 5, 3613 (2018).

[11] H.H. Pham and L. Wang, Phys. Chem. Chem. Phys. 17, 541 (2015). 